\title{
Energy dependence of ${ }^{238} \mathrm{U}$ fission yields investigated in inverse kinematics
} \author{
M. Veselsky ${ }^{9}$ \\ 1 Universidad de Santiago de Compostela, 15782 Santiago de Compostela Spain \\ 2 Centre d'Etudes Nucléaires Bordeaux-Gradignan, F-33175 Gradignan, France \\ 3 Gesellschaft für Schwerionenforschung mbH, D-64291 Darmstadt, Germany \\ 4 Heavy Ion Laboratory, University of Warsaw, PL-02-093, Poland \\ 5 WNSL, Yale University, New Haven, Connecticut 06511, USA \\ 6 Laboratory of Radiochemistry, P. O. Box 55, FI-00014 Helsinki, Finland \\ 7 Institute of Nuclear Sciences, VINCA, Belgrade 11001, Serbia \\ 8 CEA/DAM, Bruyeres-le-Chatel, 91290 Aapajon Cedex, France \\ 9 Slovak Academy of Sciences, Dubravska cesta 9, Bratislava, Slovakia
}

J. Benlliure ${ }^{1, a}$, D. Pérez-Loureiro ${ }^{1}$, H. Alvarez-Pol ${ }^{1}$, B. Blank ${ }^{2}$, E. Casarejos ${ }^{1}$, V. Fohr ${ }^{3}$, M. Gascón ${ }^{1}$, W. Gawlikowicz ${ }^{4}$, A. Heinz ${ }^{5}$, K. Helariutta ${ }^{6}$, S. Lukic ${ }^{3}$, F. Montes ${ }^{3}$, L. Pienkowski ${ }^{4}$, M. Staniou ${ }^{3}$, K. Subotic ${ }^{7}$, k. Sümmerer ${ }^{3}$, J. Taieb $^{8}$, and

\begin{abstract}
The production cross sections of neutron-rich fission residues produced in reactions induced by a ${ }^{238} \mathrm{U}$ beam impinging onto $\mathrm{Pb}$ and $\mathrm{Be}$ targets were investigated at the Fragment Separator (FRS) at GSI using the inverse kinematic technique. These data allowed us to discuss the optimum energies in fission for producing the most neutron-rich residues.
\end{abstract}

\section{Introduction}

Medium-mass neutron-rich nuclei belong to one of the most exciting regions of the chart of nuclei because of its implication in nuclear structure studies, but also in stellar nucleosynthesis. The presence of several shells at large neutron excess $(\mathrm{Z}=28, \mathrm{~N}=50$ and $\mathrm{Z}=50, \mathrm{~N}=82)$ provide an excellent ground for investigating how nuclear shells evolve with isospin [1]. Moreover, two of these shells $(Z=50, N=82)$ are also at the origin of the waiting point at $A \approx 140$ of the stellar r-process nucleosynthesis. Therefore, the production of nuclei in that region of the chart of nuclides is of utmost importance and has become a challenge for next generation radioactive beam facilities.

Although the fragmentation of neutron-rich ${ }^{136}$ Xe beams can produce some of the nuclei of interest [2], fission has been proven to be the most efficient mechanism for the production of medium-mass neutron-rich nuclei [3-6]. Indeed, all next generation radioactive beam facilities based on the ISOL or the in-flight separation techniques use the fission of ${ }^{238} \mathrm{U}$ or even ${ }^{235} \mathrm{U}$ to populate this region of the chart of nuclides. Even more, the EURISOL project proposes a two-step reaction scheme where neutron-rich fission residues would be re-accelerated and fragmented to produce medium-mass nuclei with an even larger neutron excess [7].

Together with the nature of the fissioning nucleus [8], the other parameter determining the final population of fission fragments is the energy at which fission takes place

\footnotetext{
a e-mail: j.benlliure@usc.es
}

[9]. It is well established that fission of ${ }^{238} U$ or ${ }^{235} U$ at low energies (i.e. thermal neutron induced) produces an asymmetric distribution of fission residues. By increasing the energy, the distribution becomes more symmetric but also broadens in mass number, producing heavier and lighter fission residues, and in neutron excess, leading to larger isotopic chains of fission fragments [10]. However, higher excitation energies also favored the evaporation of neutrons from the fission fragments reducing their neutron excess.

It is the aim of this work to investigate which is the optimum energy for fission in order to produce the largest variety of fission fragments with the largest possible neutron excess. This topic has been addressed by performing an experiment where the fragments produced in the fission of ${ }^{238} \mathrm{U}$ were fully identify in mass and atomic number taking advantage of the inverse kinematic technique. The role of the excitation energy was investigated by using two different targets, lead and beryllium, enhancing Coulombinduced fission at low energy and nuclear-induced fission at higher energies, respectively.

\section{The experiment}

The experiment was performed at the GSI accelerator facilities in Darmstadt. A beam of ${ }^{238} \mathrm{U}$ was accelerated by the SIS18 synchrotron at $950 \mathrm{AMeV}$. The beam was then transported until the FRagment Separator (FRS) target station. The projectile reaction residues flew forward and were analysed by the zero-degree magnetic spectrometer FRS

This is an Open Access article distributed under the terms of the Creative Commons Attribution-Noncommercial License, which permits unrestricted use, distribution, and reproduction in any noncommercial medium, provided the original work is properly cited. 


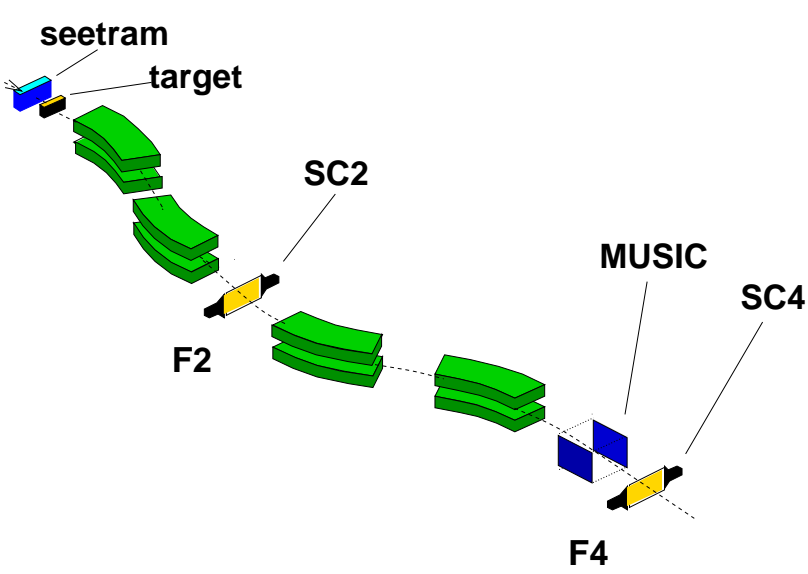

Fig. 1. Schematic layout of the Fragment Separator in the configuration used in the present experiment.

[11]. This is a two-stage achromatic spectrometer with a resolving power of $\mathrm{B} \rho / \Delta \mathrm{B} \rho \approx 1500$, a momentum acceptance of $\pm 1.5 \%$ and an angular acceptance for the central trajectory of $15 \mathrm{mrad}$ (see Fig. 1).

During the experiment the beam intensity was continuously monitored ranging from $10^{7}$ ions/s up to $5 \cdot 10^{8}$ ions/s. The two targets used in this experiment had a thickness of $1036 \mathrm{mg} / \mathrm{cm}^{2}$ for beryllium and $649 \mathrm{mg} / \mathrm{cm}^{2}$ for lead. The FRS was equipped with adequate detection systems providing the identification of the transmitted nuclei. Timeprojection chambers placed at both image planes of the spectrometer (F2 and F4 in Fig. 1) were used to determine the magnetic rigidity of the transmitted nuclei. Two fast plastic scintillators covering the full acceptance, also at both image planes, were used to measure the time of flight and then, the velocity of the nuclei. Finally, a fast ionisation chamber placed at the final image plane provided the measurement of the energy loss of the fragments with a resolution sufficient to determine their atomic number. Combining the measurements of the magnetic rigidity, velocity and energy loss we could unambiguously separate and identify in atomic and mass number all the fragments transmitted by the FRS.

Figure 2 shows a typical identification matrix obtained for a magnetic tuning of the FRS centered in ${ }^{132} \mathrm{Sn}$. As can be seen, the excellent resolving power of the spectrometer made it possible to separate and identify unambiguously the fission fragments produced in the investigated reactions. A detailed description of the experimental technique and analysis procedure can be found in Refs. [12-17].

In order to overcome the limited momentum acceptance of the FRS we performed several measurements tunning the magnetic fields of the spectrometer in order to cover a large region of magnetic rigidity. In such way we could measure the production of a large number of residual nuclei but also cover a large fraction of their momentum distribution.

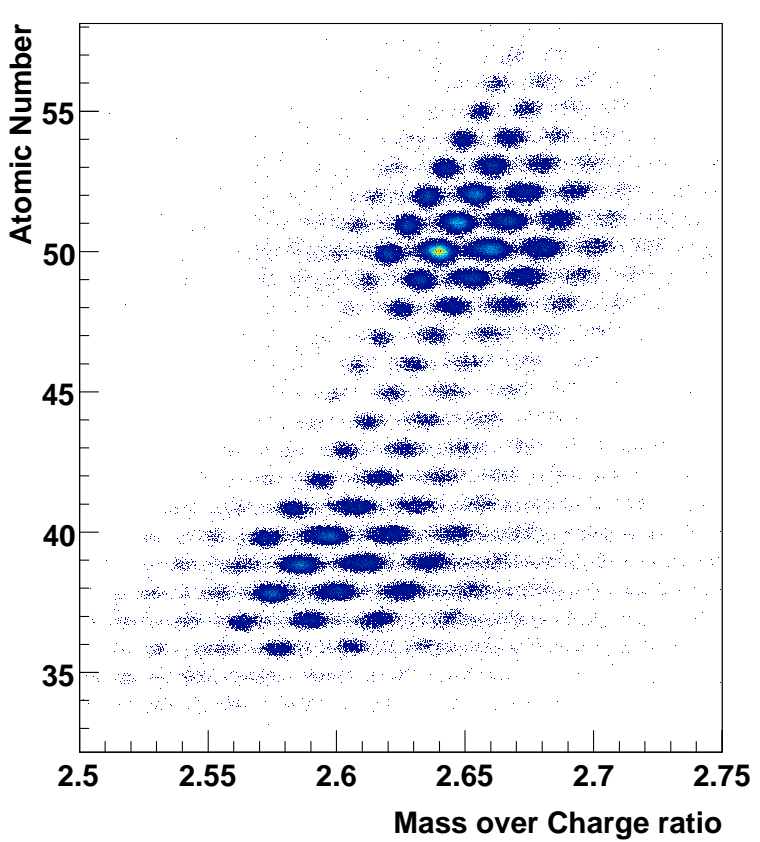

Fig. 2. Typical identification matrix for a magnetic tunning of the FRS where ${ }^{132} \mathrm{Sn}$ is transmitted along the central axis of the spectrometer.

\section{Production cross sections of fission fragments}

The fission yields obtained following the procedure described in the previous section were corrected by the losses in the different layers of matter inside the FRS, secondary reactions in the target, data acquisition dead time and optical transmission. Particular attention was paid in the accurate estimation of the optical transmission because of the limited angular acceptance of the FRS for fission fragments [18]. Finally, the corrected fission yields were normalized to the target thickness and beam current in order to determine the individual production cross sections of all fission residues identified in this experiment.

We could identify 196 and 236 fission residues produced with the beryllium and lead target, respectively. In both cases we measured relatively long isotopic chains from element $\mathrm{Z}=36$ to $\mathrm{Z}=60$ with cross sections as low as 100 $\mathrm{pb}$. The uncertainties in the measured cross sections were mostly due to the optical transmission corrections and only for the most exotic nuclei to the statistics. Typical values for these uncertainties range between $20 \%$ and $40 \%$.

The measured cross sections have been compared to data previously obtained for the same or similar reactions. In particular we have used data from the reaction ${ }^{238} \mathrm{U}+\mathrm{Pb}$ at $750 \mathrm{~A} \mathrm{MeV} \mathrm{[19],}{ }^{238} \mathrm{U}+\mathrm{Be}$ at $1000 \mathrm{~A} \mathrm{MeV} \mathrm{[5]} \mathrm{and}$ ${ }^{238} \mathrm{U}+\mathrm{d}$ at $1000 \mathrm{~A} \mathrm{MeV} \mathrm{[15].} \mathrm{The} \mathrm{first} \mathrm{set} \mathrm{of} \mathrm{data} \mathrm{agree}$ pretty well with our measurements with the lead target. Similar conclusion can be drawn from the two other sets of data when compared with our measurements with the 

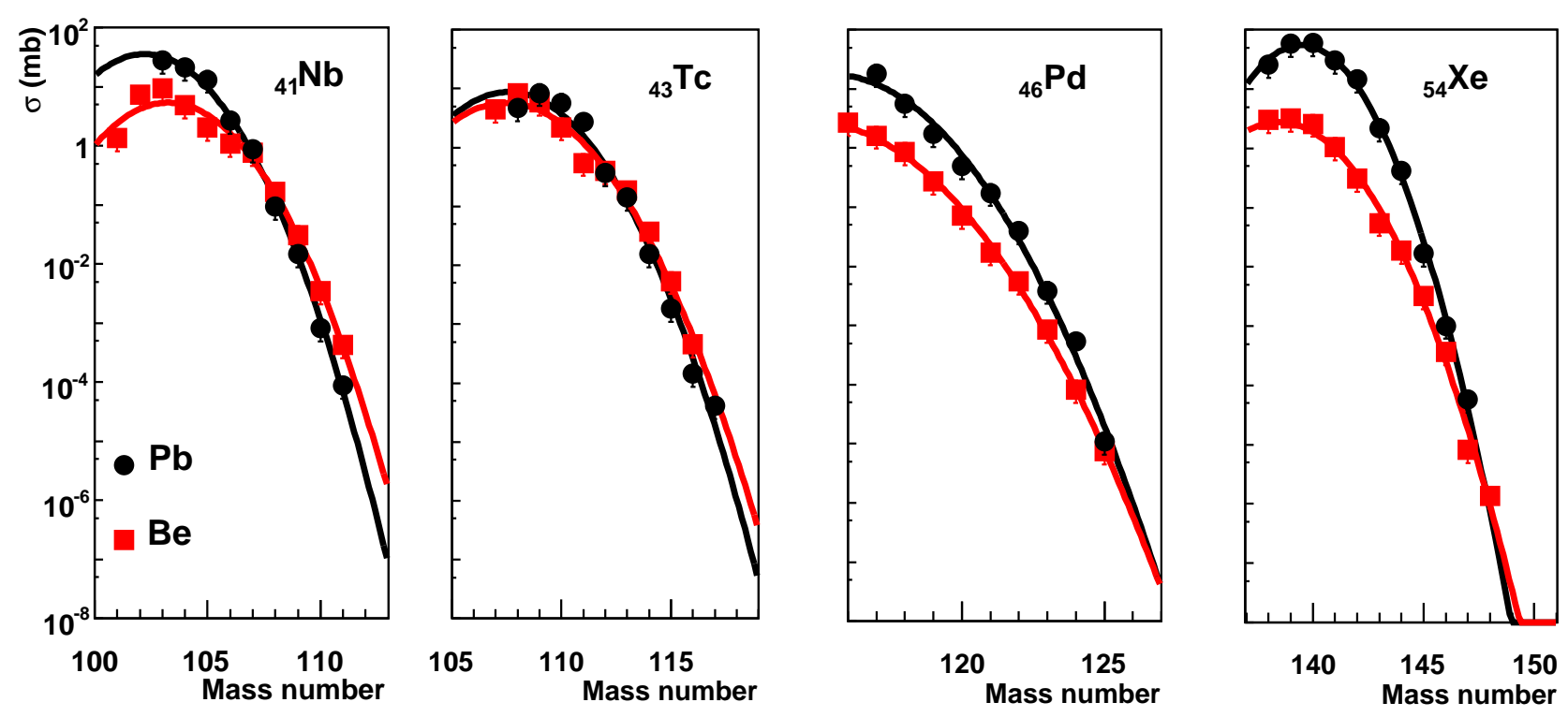

Fig. 3. Isotopic distributions of the production cross sections of fragments of niobium, tecnetium, palladium and xenon produced in the fission of ${ }^{238} \mathrm{U}$ induced by a lead (circles) and beryllium (squares) target. The solid lines correspond to parabolic fits of the measured cross sections that can be used to extrapolate the measured data.

beryllium target. In all cases, the new measurements cover a larger range in neutron excess.

In figure 3 we report the measured isotopic distributions for the production cross sections of fragments of $\mathrm{Nb}$, $\mathrm{Tc}, \mathrm{Pd}$ and $\mathrm{Xe}$ produced in the fission of ${ }^{238} \mathrm{U}$ induced by the lead (circles) and beryllium (squares) targets. As can be seen, both measurements cover a large variety of elements but in particular the production of the most neutron-rich isotopes. The production cross sections of these neutronrich fragments strongly decreases with the neutron excess. On average, for the most neutron-rich isotopes an additional neutron reduces the cross section by almost one order of magnitude.

\section{Discussion on the energy dependence of the fission yields.}

According to previous calculations [10] using the fission model described in Ref. [20], one expects a clear dependence of the fission yields with the excitation energy of the fissioning system. Fission of actinides at low energies yield a well known asymmetric mass distribution of fragments caused by the double shell closure around $\mathrm{N}=82$ and $\mathrm{Z}=50$ and a neutron excess similar to the one of the fissioning nucleus. At higher excitation energies shell effects vanish, the mass distribution becomes symmetric and fluctuations in mass asymmetry and neutron excess increase, while the average neutron excess of the final fragments is reduced because of neutron evaporation. At excitation energies above $100 \mathrm{MeV}$ one even expects the manifestation of dynamical effects in fission leading to a progressive suppression of this channel [21-23].

The present data were proposed for contributing in the determination of the optimum energy range where fission process leads to the production of the largest variety of fragments with the largest possible neutron excess. Indeed, the two target materials we have used are expected to enhance fission at low and high excitation energy, respectively. The lead target is known to enhance electromagnetic induced fission through the excitation of the GDR in ${ }^{238} \mathrm{U}$ [8]. The excitation energies at which fission takes place in this case ranges from 5 to $15 \mathrm{MeV}$. On the other hand, the beryllium target favors fragmentation reactions where fission occurs on average at higher excitation energies $\approx 50$ $\mathrm{MeV}$.

The detailed analysis of the measured cross sections confirms our expectations. Fission induced by the lead target yields an asymmetric pattern for fission fragments as reflected by the larger production cross sections of $\mathrm{Nb}$ and $\mathrm{Xe}$ isotopes with respect to the symmetric splitting (Pd). In the case of the beryllium target, the distribution becomes more symmetric with similar productions for all the elements shown in the figure.

The neutron-excess distributions show two interesting features. The maximum of the distributions obtained with both targets is not much different for the light fission residues. However, for heavy nuclei, the maximum of the distribution obtained with the lead target is more neutron-rich than the one corresponding to the distribution obtained with the beryllium target. This effect can be understood as due to the sharing of the excitation energy between the two emerging fission fragments according to their mass and their de- 
excitation by neutron evaporation. At low excitation energy neutron evaporation has a low probability, then both fission fragments keep the same neutron excess of the fissioning system. At high excitation energy the heavy fragment will gain more excitation energy than the light one. Then one expects a larger evaporation chain in the de-excitation of the heavy fragment producing a final residue with a smaller neutron excess.

The other interesting feature of the neutron excess distributions is that these distributions are wider with the beryllium target that with the lead one. Indeed, for elements lighter than palladium the production cross sections for the most neutron-rich residues obtained with the beryllium target are larger that the ones measured with the lead target. In order to extrapolate the behaviour of the production cross sections for heavy elements, we have fitted the measured distributions with a parabolic function. The extrapolation for the heavy residues confirms the larger production with the beryllium target but at larger neutron-excess.

The combined effect of these two observations leads to the following conclusions. Fission at low excitation energies produces the largest production of some specific fission fragments around ${ }^{134} \mathrm{Te}$ and ${ }^{100} \mathrm{Zr}$. However, the region around the symmetric splitting ${ }^{119} \mathrm{Pd}$ present low production yields. On the other hand, at moderate excitation energies (20 to $50 \mathrm{MeV}$ ) the mass distribution becomes more symmetric but also broadens, increasing the production yields around ${ }^{119} \mathrm{Pd}$ but also producing heavier and lighter fission fragments. Moreover, in this case the neutronexcess distribution broadens compensating the neutron evaporation effect and leading to the production of fission fragments with a larger neutron excess than obtained in low energy fission. This last conclusion is particularly valid for the light fission fragments.

\section{Conclusion}

In the present work, we have investigated the energy dependence of the production of medium-mass neutron-rich nuclei in the fission of ${ }^{238} \mathrm{U}$. For this purpose, an experiment was performed at GSI to measure the production cross sections of neutron-rich fission fragments of ${ }^{238} \mathrm{U}$ using the inverse kinematic technique. The beam accelerated at $950 \mathrm{~A} \mathrm{MeV}$ impinged into two targets $(\mathrm{Pb}$ and $\mathrm{Be}$ ) enhancing low and high energy fission. Using the high resolution magnetic spectrometer FRS, the yield distributions of fission fragments at different excitation energies was investigated, providing a full identification of the final products. We have measured production cross sections of neutron-rich nuclei from $Z=36$ to $Z=60$ down to $100 \mathrm{pb}$ for both targets. The experimental data were extrapolated in order to conclude about the fission mechanism enhancing the production of the most neutron-rich nuclei.

The data clearly show that the mass distribution of fission fragments at low excitation energy (lead target) is asymmetric, becoming more symmetric at higher excitation energies (beryllium target). The neutron excess distribution broadens with excitation energy although part of the possible gain in excitation energy is lost by neutron evaporation.
Nevertheless the data clearly indicate that neutron evaporation mostly affects the heavy residues and not so much the lighter ones. Therefore, one can conclude that fission at moderate excitation energies $(\approx 50 \mathrm{MeV})$ not only covers a larger range in elements, in particular enhancing the production of symmetric partitions around palladium, but also leads to the production of more neutron-rich nuclei, in particular for the lighter elements,

The new data clearly indicates that fission at moderate excitation energies (20-50 MeV) increases the production of extremely neutron-rich nuclei, compared to the fission at lower excitation energy, even for asymmetric cases.

This work was partially supported by the Spanish Ministry of Science and Innovation (FPA2007-62652), Xunta de Galicia and the European Commission ("Research Infrastructure Action -Structuring the European Research Area EURISOL-DS" contract n. 515768 RIDS). The EC is not liable for any use that may be made of the information contained herein.

\section{References}

1. A. Jungclaus et al., Phys. Rev. Lett. 99, (2007) 132501

2. J. Benlliure et al., Phys. Rev. C 78, (2008) 054605

3. M. Bernás et al., Phys. Lett. B 331, (1994) 19-24

4. M. Bernás et al., Phys. Lett. B 415, (1997) 111-116

5. C. Engelmann et al., Z. Phys. A 352, (1995) 351

6. P. Armbruster et al., Phys. Rev. Lett. 93, (2004) 212701

7. K. Helariutta, J. Benlliure, M. V. Ricciardi and K.-H. Schmidt, Eur. Phys. J. A 17, (2003) 181

8. K.-H. Schmidt et al., Nucl. Phys. A 665, (2000) 221

9. P. Armbruster, Nucl. Phys. A 140, (1970) 385-399

10. J. Benlliure and K.-H. Schmidt, Nucl. Phys. A 746, (2004) 281

11. H. Geissel et al., Nucl. Instr. and Meth. B 70, (1992) 286-297

12. J. Benlliure et al., Nucl. Phys. A 683, (2001) 513

13. M. Bernás et al., Nucl. Phys. A 725, (2003) 213

14. B. Fernández-Dominguez et al., Nucl. Phys. A 747, (2005) 227

15. J. Pereira et al., Phys. Rev. C 75, (2007) 014602

16. T. Enqvist et al., Nucl. Phys. A 686, (2001) 481

17. T. Enqvist et al., Nucl. Phys. A 703, (2002) 435

18. J. Benlliure, J. Pereira and K.-H. Schmidt, Nucl. Instr. and Meth. A 478, (2002) 493

19. T. Enqvist et al., Nucl. Phys. A 658, (1999) 47

20. J. Benlliure et al., Nucl. Phys. A 628, (1998) 458-478

21. J. Benlliure et al., Nucl. Phys. A 700, (2002) 469

22. B. Jurado et al., Phys. Rev. Lett. 93, (2004) 072501

23. J. Benlliure, E. Casarejos, J. Pereira and K.-H. Schmidt, Phys. Rev. C 74, (2006) 014609 